\title{
Plasmonic Polymers Unraveled Through Single Particle Spectroscopy
}

Liane S. Slaughter ${ }^{a b c}$, Lin-Yung Wang ${ }^{a b \uparrow}$, Britain A. Willingham ${ }^{a b}$, Jana M. Olson ${ }^{a b}$, Pattanawit Swanglap $^{a d b}$, Sergio Dominguez-Medina ${ }^{a b}$, Stephan Link ${ }^{a b e} *$

${ }^{a}$ Department of Chemistry, Rice University, 6100 Main St., Houston, TX, 77005, USA

${ }^{b}$ Laboratory for Nanophotonics, Rice University, 6100 Main St., Houston, TX, 77005, USA

${ }^{c}$ Present address: California NanoSystems Institute, University of California at Los Angeles, 570 Westwood Plaza, Los Angeles, CA, 90095, USA

${ }^{d}$ Present address: Department of Chemistry, Silpakorn University, Nakhon Pathom, Thailand ${ }^{e}$ Department of Electrical and Computer Engineering, Rice University, 6100 Main St., Houston, TX, 77005, USA

"Authors contributed equally

*Address correspondence to slink@rice.edu

\section{TOC GRAPHIC}

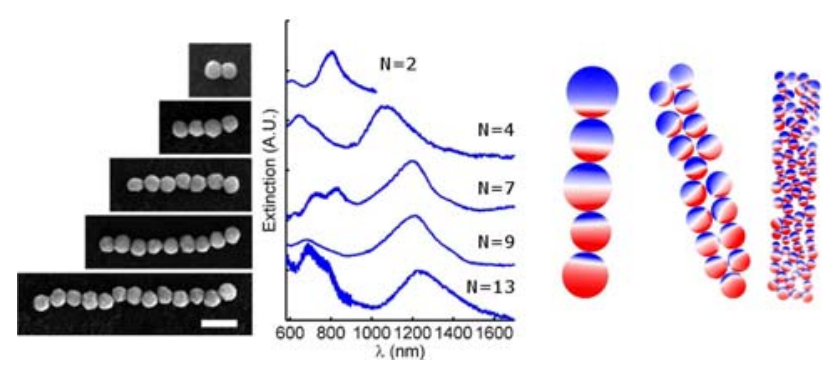

KEYWORDS: Gold nanoparticles, surface plasmon resonance, plasmon coupling, super- and sub- radiant modes, nanoparticle chains, single particle spectroscopy 


\begin{abstract}
Plasmonic polymers are quasi one-dimensional assemblies of nanoparticles whose optical responses are governed by near-field coupling of localized surface plasmons. Through single particle extinction spectroscopy correlated with electron microscopy, we reveal the effect of the composition of the repeat unit, the chain length, and extent of disorder on the energies, intensities, and line shapes of the collective resonances of individual plasmonic polymers constructed from three different sizes of gold nanoparticles. Our combined experiment and theoretical analysis focuses on the superradiant plasmon mode, which results from the most attractive interactions along the nanoparticle chain and yields the lowest energy resonance in the spectrum. This superradiant mode redshifts with increasing chain length until an infinite chain limit, where additional increases in chain length cause negligible change in the energy of the superradiant mode. We find that, among plasmonic polymers of equal width comprising nanoparticles with different sizes, the onset of the infinite chain limit and its associated energy are dictated by the number of repeat units and not the overall length, of the polymer. The intensities and linewidths of the superradiant mode relative to higher energy resonances, however, differ as the size and number of nanoparticles are varied in the plasmonic polymers studied here. These findings provide general guidelines for engineering the energies, intensities, and line shapes of the collective optical response of plasmonic polymers constructed from nanoparticles with sizes ranging from a few tens to one hundred nanometers.
\end{abstract}

\title{
INTRODUCTION
}

Strong near-field coupling between localized surface plasmons supported by metallic nanoparticles (NP) enables line shape engineering of the absorption and scattering spectrum in 
the visible to near-infrared region. ${ }^{1-7}$ To explain the collective behavior of coupled surface plasmons, several different concepts have been inspired by well-established theories developed in physics and chemistry, such as plasmon hybridization. ${ }^{4,6}$, group theory ${ }^{8}$, Fano resonances ${ }^{2,9}$, and superradiance. ${ }^{10}$ Similarly, the assembly process of gold nanorods has been successfully described by polymer growth kinetics derived for polymerization reactions. ${ }^{11-13}$ In the spirit of such analogies, plasmonic polymers are quasi one-dimensional NP assemblies with collective optical properties that can be tuned by changing the composition of the monomer repeat unit. The overall optical properties of NP chains have been shown to depend on the composition of the repeating monomer, establishing a property that is fundamental to the definition of a polymer., ${ }^{14-16}$ Furthermore, much like a conjugated organic polymer, ${ }^{17-20}$ an infinite chain limit defines the resonance maximum of the lowest energy plasmon mode, which results from the most attractive interaction of the individual NP dipoles aligned in phase along the longitudinal chain axis and is referred to as the superradiant mode. ${ }^{3,15,21-24}$ Finally, distributions in NP size and arrangement, often inherent to experimental systems, modify the collective optical response, but the effect of such disorder is overall small for the energy of the superradiant mode in large NP assemblies and generally only leads to changes in relative peak intensities and linewidths., ${ }^{34,25-28}$ This behavior parallels the insensitivity of the optical spectra measured for conjugated polymers having a variety of chain lengths and conformations as their optical properties are governed by localized chromophores consisting of a minimum number of repeat units. ${ }^{17,19,20,29-31}$

Both individual NPs and simple assemblies of NPs, or plasmonic molecules, can serve as the repeating monomer unit in plasmonic polymers. Single particle studies have proven that the sizes, shapes, compositions, and interparticle spacings within a dimer of plasmonic NPs, the simplest plasmonic molecule, sensitively affect the energy of the resulting coupled plasmon 
modes and the amplitude of the enhanced electric field between the NPs. ${ }^{1,-7,32-39}$ More complex plasmonic molecules composed of more than two NPs present even more diverse avenues for tuning the excitation energy, amplitude, and distribution of the enhanced localized electric field and for engineering spectral features such as Fano resonances and magnetic modes that are otherwise absent in simpler assemblies. ${ }^{2,7,40,41}$ Plasmonic molecules have been fabricated by electron beam lithography and chemical assembly methods, where the latter add further variety due to the potential to assemble many NP structures in parallel..$^{2,11,41-43}$ At the expense of increased disorder, bottom-up assembly methods yield smaller interparticle spacings that maximize near-field coupling and even allow one to tune interparticle distances and relative orientations, i.e. bond lengths and bond angles. ${ }^{12,40,44,45}$ Because of the complexity involved in transitioning from plasmonic molecules to polymers, basic guidelines for the relationships between plasmonic polymer geometry and collective optical response are necessary for rational design and application of these metallic nanostructures.

The ability of linear chains of plasmonic NPs to function as nanoscale optical antennas and waveguides has already stimulated detailed investigations into how length, interparticle gaps, and disorder affect the energies, oscillator strengths, and linewidths of coupled plasmon modes in chains of Ag and Au NPs. ${ }^{21,46-49}$ As NPs are added successively to the length of the chain, the superradiant mode initially shows a continuous redshift, which then saturates as an infinite chain limit is reached. To summarize a body of literature, as long as $2.0<D / r \leq 2.5$, where $D$ is the center-to-center distance between the NPs and $r$ is the NP radius, the energy of the lowest energy peak plateaus for a number of repeat units, $N_{L}$, between 5 and 10, even though a range of $r(5-55 \mathrm{~nm})$ and interparticle gaps $(0.5-30 \mathrm{~nm})$ has been investigated. ${ }^{21,23,25,47,48,50-}$ 52 The interaction strength between surface plasmons is significantly reduced though when $D / r$ 
increases from 2.01 to 2.1 , as investigated in detail both experimentally and through simulations for the smallest chain lengths of dimers and trimers. ${ }^{32,}{ }^{53-55}$ Due to this decrease in coupling, saturation of the redshifting for the lowest energy longitudinal chain mode is predicted to occur at chain lengths as short as 7 NPs and at shorter wavelengths as the interparticle gap increases. ${ }^{3,}$ ${ }^{50-52}$ For example, the resonance maximum $\lambda_{\max }$ of the superradiant longitudinal mode in the extinction spectra of lithographically fabricated Au disks with $r=25 \mathrm{~nm}$ and $D=75 \mathrm{~nm}$ continues to redshift until $N_{L} \sim 9 \mathrm{NPs}$, but the total shift between the single NP resonance and $\lambda_{\max }$ of the lowest energy chain mode was only $\sim 12 \mathrm{~nm}^{47}$

Most recently, the groups of Mulvaney and Reinhard used dark-field scattering single particle spectroscopy to demonstrate the onset for the saturation of the redshifting in the superradiant plasmon mode for individual chains that were composed of Au NPs with diameters of 64 and $40 \mathrm{~nm}$, respectively, and were one NP wide with a maximum length of $7 \mathrm{NPs} .^{3,25}$ Accompanying theory highlights the sensitivity of the optical response on the interparticle gap as increasing $D / r$ decreases the overall redshift achieved upon reaching the infinite chain limit. Similarly, Kumacheva and coworkers correlated ensemble spectra with electron microscopy images to characterize the dependence of the plasmon resonance redshift in plasmonic polymers comprising nanorods on the average aggregation number, and also found that plasmon coupling and the corresponding spectral shift decreases with increasing $D / r{ }^{15}$

The studies of Mulvaney and Reinhard are among the few experiments that test the significance of important geometric parameters such as NP size and interparticle distance on the response of individual NP chains with $D / r<2.05$, but each study is limited to $N_{L} \leq 7$, one average NP size, and chains of only one NP wide. In addition, the consequences of disorder are beyond the scope of those studies. Increasing the number of NPs in experimentally assembled 
chains also increases the distribution of sizes, shapes, and off-linear NP placements. Scheurer et al., Esteban et al., and Rüting have introduced disorder parameters to their theoretical investigations and have shown that departure from well-ordered linear arrangements modulates the relative intensities of the superradiant and higher order modes in the spectra of individual chains. ${ }^{27,56,57}$ Although Esteban et al. simulated NP chain geometries specifically inspired by aggregates of Au NPs, they computationally randomized and averaged the chain configurations to match optical spectra recorded in solution. The dependence of the plasmonic response on geometric parameters in the presence of experimentally inherent disorder for individual chains therefore still needs to be elucidated further.

We previously explored linear chains of $47 \mathrm{~nm}$ Au NPs and showed that constructing a plasmonic polymer using dimers of Au NPs, instead of single Au NPs, as the repeating monomer causes the superradiant mode to redshift further even if the number of repeat units, $N_{L}$, and overall physical length, $L_{\text {Tot }}$, are similar. ${ }^{14}$ Theoretical analysis revealed that for both double and single column chains, the superradiant mode not only reaches an infinite chain limit for $N_{L}=5$ 10 , consistent with earlier studies, ${ }^{27,50,52}$ but also redshifts due to plasmonic interactions as well as phase retardation as $L_{T o t}$ increases. ${ }^{50,51}$ As the latter effect also depends on the width of the plasmonic structure when $N_{L}$ and $L_{T o t}$ are similar and considering that the double column chain was twice as wide compared to the single column chain, it was not possible to evaluate the role of $N_{L}$ vs. $L_{T o t}$ in defining the resonance energies of the superradiant mode for plasmonic polymers with different repeat units. We therefore present here a comparison between plasmonic polymers that more rigorously unravel the influence of $N_{L}$ and $L_{T o t}$ on the extinction spectra of plasmonic polymers, with particular emphasis on the superradiant mode. 
The current study provides three comparisons of plasmonic polymers, which all have a similar width of $\sim 100 \mathrm{~nm}$ and vary $N_{L}, L_{T o t}$, and the type of repeat unit, as illustrated schematically in Fig. 1. The repeat units used here are one large NP with diameter $d=94( \pm 8)$ $\mathrm{nm}$, a dimer of medium NPs with $d=47( \pm 4) \mathrm{nm}$, and a linear tetramer of small NPs with $d=25$ $( \pm 3) \mathrm{nm}$. Using the largest NPs, we first establish experimental agreement with the predicted infinite chain length at $N_{L}=5-10 \mathrm{NPs}$ for this plasmonic polymer by varying $N_{L}$ up to 13 , significantly beyond what has been reported before for single particle studies. ${ }^{25,27,40,52}$ Our direct observation of the infinite chain limit eliminates the need to establish it through simulations. Next, we set $L_{T o t}$ and the width constant (Fig. 1b) and allow $N_{L}$ to vary from 5 large NPs, $\sim 10$ dimers of medium NPs, to $\sim 20$ tetramers of the smallest NPs. Finally, we compare chains of the same $N_{L}$ and width, but varying $L_{T o t}$ due to the different NP size (Fig. 1c). Simulated extinction spectra of chains with NP arrangements taken from scanning electron microscopy (SEM) images as well as ideal closely packed assemblies aid in the interpretation of the experiments and furthermore give insight into how disorder in addition to the geometric parameters outlined in Fig. 1 modifies the energies, intensities, and shapes of the spectral features. Our results show that the energy of the superradiant mode of plasmonic polymers consisting of NPs of different sizes but overall similar widths is determined by $N_{L}$ and not $L_{T o t}$, once the infinite chain limit has been reached. Substituting large NPs for monomers comprising assemblies of smaller NPs, however, decreases the intensity and broadens the width of the resonances. 


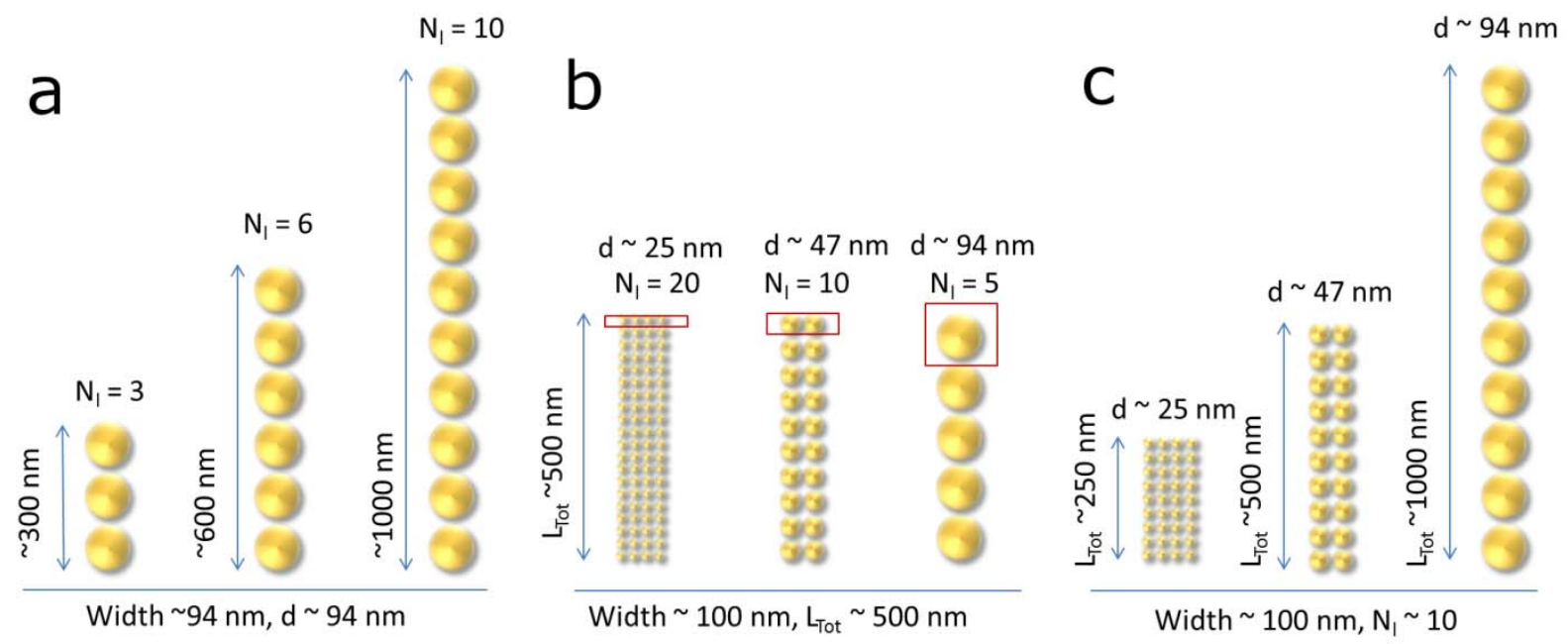

Fig. 1 Comparisons among plasmonic polymers tested experimentally here. The width is held constant in all situations. (a) The optical properties of chains with varying $N_{L}$ reveal an infinite chain limit. These chains vary in both $N_{L}$ and $L_{T o t}$, however, obfuscating the effects of plasmon coupling over a different number of NPs $N_{L}$ and retardation with increasing $L_{T o t}$. $L_{T o t}$ is therefore held constant in (b) to isolate the role of $N_{L}$ for different types of monomers, indicated in the red boxes. In (c), the number of repeat units is held constant while $L_{T o t}$ varies due to the different sizes of the constituents.

\section{EXPERIMENTAL SECTION}

\section{Sample Preparation}

Plasmonic polymers of Au NPs were assembled via a combination of the Marangoni effect and capillary action as described previously. ${ }^{46}$ Glass substrates coated with indium tin oxide (ITO, Delta Technologies) were trimmed to rectangular chips of less than $1 \mathrm{~cm} \mathrm{x} 1 \mathrm{~cm}$ and cleaned by incubating them for 30 minutes at $45^{\circ} \mathrm{C}$ in a 1:4:20 $\mathrm{NH}_{4} \mathrm{OH}: \mathrm{H}_{2} \mathrm{O}_{2}: \mathrm{H}_{2} \mathrm{O}$ mixture followed by sonicating 3 times in deionised water for 15 minutes each. The chips were then cleaned in an $\mathrm{O}_{2}$ plasma and irradiated with $365 \mathrm{~nm}$ UV light for approximately 90 seconds. 
Poly(methyl methacrylate) (PMMA, A4 $M_{W}=495 \mathrm{k} \mathrm{g} / \mathrm{mol}$, Microchem) in anisole was spin coated onto the chips at $3000 \mathrm{rpm}$ for 40 seconds and soft baked at $180^{\circ} \mathrm{C}$ for 90 seconds before mounting the chip to the sample stage of a SEM. Trenches were created in the PMMA resist using electron beam lithography performed on a JEOL 6500 SEM operating at $30 \mathrm{kV}$ and equipped with the Nabity Pattern Generation System. Patterns were developed by placing the chip in a 1:3 mixture of methyl-isobutylketone:isopropanol for 25 seconds and then in a stop bath of isopropanol for several minutes, followed by blow drying with compressed $\mathrm{N}_{2}$.

Au NPs capped with sodium citrate were deposited onto the substrates within 48 hours of completing the lithography. The Au NPs (NanoPartz) had diameters of $25( \pm 3) \mathrm{nm}, 47( \pm 4)$, and $94( \pm 8) \mathrm{nm}$ as determined by transmission electron microscopy (TEM, JEOL 1230, Supplementary Information Fig. S1). A $5 \mu 1$ drop of Au NPs in an aqueous solution with an optical density of $\sim 1$ was placed on the patterned region of the chip. NPs filled the trenches as the meniscus of the drop passed over the trenches. The drop was allowed to dry completely. The presence of filled trenches was first confirmed by SEM in a FEI Quanta 400 ESEM2 operating at $20 \mathrm{kV}$ in wet mode at low magnification. Then the resist was lifted-off by immersing the chip in acetone for several minutes with brief sonication and the samples were finally dried with compressed $\mathrm{N}_{2}$.

\section{Correlated Electron Microscopy and Single Particle Optical Spectroscopy}

SEM images were acquired using the FEI Quanta 400 ESEM2 operating at $20 \mathrm{kV}$ in high vacuum mode. SEM was first performed before optical spectroscopy at a magnification $\leq$ 40,000X to select the chains with closely packed NPs and the desired dimensions, i.e. width, $N_{L}$, and $L_{T o t}$. To avoid melting especially the smallest Au NPs with the electron beam and to 
minimize effects due to interaction of organic contaminants with the electron beam, high magnification SEM to resolve the geometry of individual chains was performed after optical spectroscopy.

Broadband extinction spectra of Au NP chains were acquired in transmission geometry as previously described. ${ }^{58}$ The same PMMA resist was used to glue the chip to a 24 x $50 \mathrm{~mm}$ glass cover slide (Fisher) that was mounted on a nanopositioning piezo stage (Physik Instrumente). Light from a halogen lamp (Newport) was coupled into a fiber bundle mounted above the sample for normal incidence excitation, which minimizes differences regarding the spectral dependence of plasmon modes on the direction of the excitation light ${ }^{2}$ and enables easier comparison with calculations. The transmitted light was collected by a 50x/0.8 EC-Epiplan Neofluar air-spaced objective in an inverted microscope (Zeiss) and, after passing through a $50 \mu \mathrm{m}$ pinhole placed in the first image plane outside the microscope, detected by an avalanche photodiode (APD, Perkin Elmer). By moving the scanning stage, an image was obtained enabling us to locate the Au NP chain of interest. The light was then redirected toward a Si CCD camera (Princeton Instruments) attached to a spectrograph (Princeton Instruments) to record extinction spectra in the wavelength range of 500 to $950 \mathrm{~nm}$. To acquire spectra from $900-1700 \mathrm{~nm}$, the light was focused onto the end of a $100 \mu \mathrm{m}$ diameter fiber that directed the light toward a spectrometer (Andor Technology) equipped with an InGaAs array detector (Andor Technology). The extinction spectrum was calculated according to:

$$
\text { Extinction }=-\log \left(\frac{I-I_{d c}}{I_{0}-I_{d c}}\right)
$$


where $I$ is the transmitted light intensity with a nanostructure in the observation volume, $I_{0}$ is the intensity in a background region without Au NPs, and $I_{d c}$ are the dark counts measured with the lamp switched off.

The scattering spectra of individual 47 and $94 \mathrm{~nm}$ Au NPs, the dimer of $94 \mathrm{~nm}$ Au NPs in Fig. a, and the linear tetramer of $25 \mathrm{~nm}$ Au NPs in the inset of Fig. 3c were acquired by darkfield spectroscopy in reflection geometry on the same microscope used to take extinction spectra. Excitation light from a halogen lamp was focused onto the sample using the same 50x/0.8 objective and the scattered light was collected in a reflected light geometry. The sample was again raster scanned to form an image using the APD, then repositioned to the Au NP chain of interest, and the light was redirected to the spectrograph. The spectra were normalized to the lamp spectrum acquired using a white light standard (Labsphere SRS-99-010) and corrected for dark counts. Spectra of single $25 \mathrm{~nm}$ Au NPs on the ITO coated substrates used throughout this study could not be measured because their scattering intensity was too weak due to the limited sensitivity of reflected light dark-field spectroscopy and the increased background because of the roughness of the ITO substrate.

\section{Generalized Mie Theory Calculations}

The generalized Mie theory (GMT) code we used previously was improved based on the T-matrix Fortran code for parallel computer clusters provided by Mackowski and Mishchenko. ${ }^{14}$

${ }^{59}$ Mie theory provides the analytical solution to Maxwell's equations for a single sphere. The scattered and incident fields are represented as an expansion of vector spherical harmonics with the appropriate weighting coefficients. Analyzing the energy flow provides analytical equations for the optical cross-sections. The GMT extends this solution to $N$ spheres, where scattered 
fields from neighboring spheres act as further excitation. The scattering coefficients for each of the $N$ spheres is solved by considering a system of linear equations. ${ }^{60}$

The electric fields and optical cross-sections presented here were calculated via the GMT using input parameters from the geometry of the Au NP chains provided by SEM. The geometry of each assembly was reproduced by an image analysis algorithm based on the Hough transform, allowing the user to determine both the diameter and location of individual constituent NPs. ${ }^{61}$ In all calculations, the minimum surface-to-surface separation was $0.5 \mathrm{~nm}$, ensuring an optical response that can be described by solely capacitive near-field coupling of the plasmon modes without quantum effects. ${ }^{50,62}$ Although our previous calculations used a minimum separation of $1 \mathrm{~nm},{ }^{14} 0.5 \mathrm{~nm}$ gaps with 23 multipoles provided in general overall better agreement between experiment and theory. For consistency among all simulations, we applied this minimum separation to the calculation of the single and double column chains of 47 Au NPs reported before ${ }^{14}$ and presented here in Figs. $5 \mathrm{~b}$ and $5 \mathrm{e}$ as well as Figs. $6 \mathrm{~b}$ and $6 \mathrm{e}$. Interparticle distances below a few nanometers cannot be resolved by SEM and therefore a range of $0.5-1.0 \mathrm{~nm}$ is a good estimate for the minimum edge-to-edge distance between closely packed Au NPs in the chains fabricated here.

Likewise, discerning from the SEM images the sizes, positions, and separations among every NP in chains consisting of closely packed $25 \mathrm{~nm}$ Au NPs proved to be difficult without using higher magnifications that would have damaged the sample during SEM imaging. We found that randomized arrangements of Au NPs with different diameters of $d=23 \mathrm{~nm}$ and $d=27$ $\mathrm{nm}$ provided suitable models to represent the measured optical response for the $25( \pm 3) \mathrm{nm} \mathrm{Au}$ NP chains as long as the overall chain geometry and number of Au NPs was the same as determined from SEM. The two sizes of 23 and $27 \mathrm{~nm}$ fall within the distribution of Au NP 
sizes measured by TEM for this sample and were chosen to increase the filling efficiency compared to spheres of uniform size. A collision-driven molecular dynamics algorithm was used to produce the random Au NP positions. ${ }^{63,64}$

Unpolarized spectra were calculated by averaging the in-plane polarization components of the normal incident excitation light. The material response of each NP was modeled using the bulk response of gold, with the complex dielectric function taken from the tabulated values by

Johnson and Christy. ${ }^{65}$ An effective medium dielectric constant for NPs on an ITO substrate and surrounded by air was obtained by finding the best agreement between measured and simulated scattering spectra for individual $94 \mathrm{~nm}$ Au NPs deposited on the same ITO glass substrates. The measured scattering spectrum of a single Au NP was fit to a Lorentzian curve and the medium dielectric constant in the simulations was adjusted so that the maximum peak wavelength and linewidth of the calculated and measured spectra matched (Supplementary Information Fig. S2). A refractive index of 1.32 provided the best agreement between the simulations and the experiment and was therefore applied to all GMT simulations.

\section{RESULTS AND DISCUSSION}

The extinction spectra in Fig. 2a, representing the experimental realization of Fig. 1a with $94 \mathrm{~nm} \mathrm{Au} \mathrm{NPs,} \mathrm{show} \mathrm{that} \mathrm{the} \mathrm{resonance} \mathrm{maximum} \lambda_{\max }$ of the lowest energy plasmon mode redshifts with increasing $N_{L}$ until an infinite chain limit is reached. Beyond the infinite chain limit, successive addition of NPs to the length of the chain does not significantly modify the energy of this resonance. Consistent with simulations using the GMT for linear chains of uniform NP size, the measured trend shown in Fig. $b$ demonstrates that $\lambda_{\max }$ of the superradiant 
mode plateaus around $N_{L} \sim 5-10$. The infinite chain limit is thus reached at this number for these $94 \mathrm{~nm} \mathrm{Au} \mathrm{NPs,} \mathrm{and} \mathrm{the} \mathrm{small} \mathrm{differences} \mathrm{in} \mathrm{the} \mathrm{measured} \lambda_{\max }$ compared to the simulated trend are attributed to the distribution of NP sizes, shapes, and interparticle gaps as well as minor off-linear alignments of the NPs.

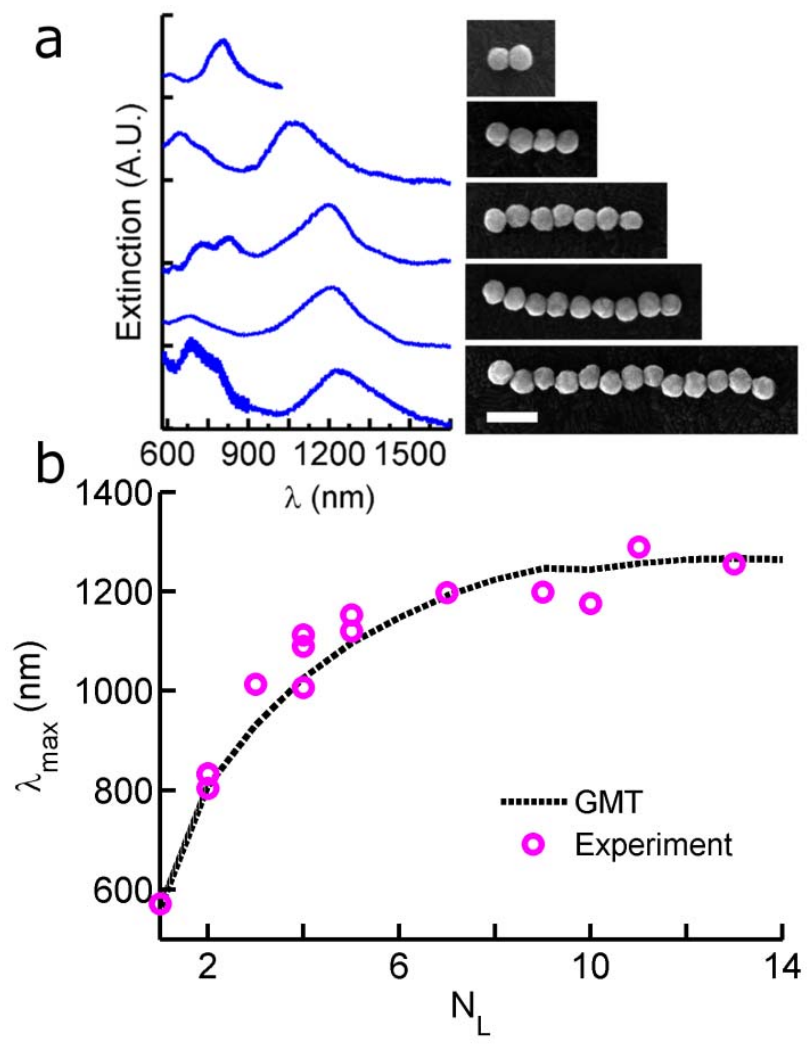

Fig. 2 Dependence of the spectral response of plasmonic polymers on the number of repeat units $N_{L}$. (a) Broadband extinction spectra of chains consisting of $94 \mathrm{~nm}$ Au NPs with varying $N_{L}$ and corresponding SEM images. The scale bar represents $200 \mathrm{~nm}$. For $N_{L}=2$, the dark-field scattering spectrum is shown as the coupled mode is still within the spectral response of the CCD camera. All spectra were normalized to the peak of the lowest energy mode. (c) Measured and simulated scaling of the peak wavelength of the superradiant mode with chain length expressed as $N_{L}$.

The physical mechanisms giving rise to the lowest energy coupled chain mode, its redshift, and the eventual saturation of the redshift with increasing $N_{L}$ are quite complex and have not been discussed in great detail compared to simply observing these trends. We give here a discussion of the different, tightly interrelated factors that all contribute to the trend observed in Fig. 2 for the lowest energy mode of a one NP wide chain, such as the influence of superradiance 
and retardation, in addition to near-field plasmon coupling. For chains having lengths shorter than $\lambda_{\max } / 2$, the intensity of the lowest energy mode dominates the optical spectrum for interparticle gaps smaller than the radii of the individual NPs and arises from the in phase interaction of the individual NP dipoles along the longitudinal axis. ${ }^{21,47,50-52}$ Nearest neighbor coupling alone cannot account for the saturation of the redshift with increasing $N_{L}$ though, and therefore interactions among non-neighboring NPs also contribute within the limits of the superradiant coherence length. ${ }^{66}$ The dominant intensity of the lowest energy mode is the manifestation of superradiance, where coherent excitation of two or more radiating particles causes their dipole moments to become correlated. Due to this coherence, they radiate more strongly than the sum of their individual dipole moments would otherwise predict. The coherence length is defined by half the wavelength of light at the resonant wavelength of the superradiant mode. Thus, as more NPs are added to the length of the chain in Fig. 2, the lowest energy mode broadens and gains intensity within the superradiant coherence length.

Retardation causes the resonance energy of the lowest-energy mode, $\lambda_{\max }$, to decrease with increasing NP size and overall dimensions of the chain. ${ }^{49,67-69}$ Once the size of a nanostructure surpasses the quasistatic size regime $(\lambda \gg r$, i.e. $\sim 20 \mathrm{~nm})$, phase retardation depolarizes the electric field of the incident light over the dimensions of the NP. ${ }^{69-71}$ Radiation damping, which scales with $1 / r^{2}$, broadens the width of the plasmon resonance, and dynamic depolarization, which scales with $1 / r^{3}$, causes all resonant modes to redshift. Thus, the lowest energy mode of individual as well as assembled NPs redshift and broaden substantially with increasing size for dimensions outside the quasistatic limit due to retardation.

Apart from the size dependence of superradiance and retardation, increasing the size of the constituent NPs in one-dimensional chains directly affects interparticle plasmon coupling by 
increasing the amplitude of the fields scattered by the individual NPs. The scattering cross sections of individual NPs with diameters $\leq 100 \mathrm{~nm}$ increase with $V^{2}$, where $V$ is the NP volume. $^{68,70,72}$ These scattered fields result in further constructive and destructive interference for different plasmon modes. ${ }^{49,52,68}$

Finally, in addition to the general increase in plasmon coupling for smaller interparticle distances, higher order plasmon modes of the individual NPs gain strength and couple with higher order and dipole modes of the neighboring NPs. ${ }^{50}$ Even if the sizes of the individual NPs fall within the quasistatic regime where only the dipole mode contributes to the optical response of an individual NP, closely spaced assemblies of small NPs still require consideration of nearfield coupling via higher order modes. Theoretical simulations have furthermore shown that to a significantly greater extent, these higher order modes of the constituent NPs must be included to correctly model the collective chain resonances as $D$ decreases. ${ }^{21,50,51}$ Compared to the lowest energy mode, the intensities and wavelengths of peaks corresponding to these higher order coupled modes are more easily modulated due to symmetry breaking in experimental assemblies, described more thoroughly in the ensuing discussion. ${ }^{14,56}$

In Fig. 2, $N_{L}$ and $L_{T o t}$ simultaneously increase as successive NPs are added to the length of the chain. Unraveling the contributions to the spectral shape from plasmon coupling in plasmonic polymers with different repeat units requires us to hold the length and width of the assembly constant, as both retardation and the superradiant coherence length depend on the absolute dimension of the nanostructure, and not the number of NPs. ${ }^{49,} 68,70,73$ Fig. 3 experimentally tests Fig. 1b, comparing chains comprising three different kinds of monomers with the same total length, $L_{T o t} \sim 500 \mathrm{~nm}$, and width $\sim 100 \mathrm{~nm}$. We have previously shown that changing the width of the plasmonic polymer by replacing a single NP repeat unit with a dimer 
affects the energies of the superradiant mode due to a combination of plasmon coupling and retardation. ${ }^{14}$ The width of the different plasmonic polymers is therefore held constant for all studies here. Fig. 3 shows spectra of plasmonic polymers, where $L_{T o t}$ of each chain is conserved by changing the composition from 5 individual $94 \mathrm{~nm} \mathrm{Au} \mathrm{NPs} \mathrm{(chain} \mathrm{5N1)} \mathrm{to} 9$ dimers of $47 \mathrm{~nm}$ Au NPs (chain 9N2) or 20 linear tetramers of $25 \mathrm{~nm}$ Au NPs (chain 20N4). According to Fig. 2, chain $5 \mathrm{~N} 1$ is at the onset of the infinite chain limit defined by $N_{L}$, while the other two chains despite their smaller NPs are expected to have also reached or even exceeded the infinite chain limit based on previous studies of chains with NPs of different diameters. ${ }^{27,50,52}$

The spectra in Fig. 3, especially those for 5N1 and 9N2, are remarkably similar, and we conclude that substitution of repeat units does not significantly alter the energy of the lowest energy mode for constant $L_{T o t}$ and width, considering the infinite chain limit has been reached. The superradiant modes from the chain of 5 single NPs (chain 5N1) in Fig. 3a and the chain of 9 dimers (chain 9N2) in Fig. 3b occur at 1155 and $1190 \mathrm{~nm}$, respectively. The significant redshift between these lowest energy modes and the resonant dipole modes of their repeat units derives from both retardation and plasmon coupling as discussed above. The fact that the number of repeat units in chain $9 \mathrm{~N} 2$ has nearly doubled from chain $5 \mathrm{~N} 1$ while the absolute chain length of $\sim 500 \mathrm{~nm}$ has remained the same, suggests that retardation effects dominated by the overall plasmonic polymer dimensions mainly determine the energy of the superradiant mode. Plasmon interactions over more NPs, however, lead to broadening of the resonance, as indeed the FWHM of $275 \mathrm{~nm}$ for chain $9 \mathrm{~N} 2$ is larger than the FWHM of $230 \mathrm{~nm}$ for chain $5 \mathrm{~N} 1 .^{51}$ The ensuing discussion below on the simulated spectra of these chains will address in more detail the interplay of retardation, plasmon coupling, and superradiance on the position and shape of the lowest energy collective plasmon resonance. 
Compared to chains $5 \mathrm{~N} 1$ and 9N2, the extinction spectrum in Fig. $3 \mathrm{c}$ of the chain of 20 tetramers (chain 20N4), which also has $L_{T o t} \sim 500 \mathrm{~nm}$, similarly has a lowest energy mode near $1200 \mathrm{~nm}$. The significant redshift from the dipole resonant mode at $725 \mathrm{~nm}$ of the repeat unit shown in the inset of Fig. 3c implicates significant coupling over many repeat units in chain 20N4. The lowest energy resonance of chain $20 \mathrm{~N} 4$ is, however, severely broadened, and it is likely that the increased width is not just due to additional plasmon coupling over more NPs, but can also be attributed to the superposition of different resonant modes arising from many localized short range and long range plasmonic interactions within the chain and polarized in both longitudinal and transverse directions. ${ }^{14}$ Whilst the $25 \mathrm{~nm}$ NPs can easily pile into multiple layers on top of the substrate, multi-layered regions of chains are easily identified by their increased contrast in SEM compared to single layer chains, as seen in Fig. S7, and excluded from our analysis and discussion. Even with the analysis restricted to a single layer of NPs, the larger number of NPs in chain $20 \mathrm{~N} 4$, compared to chains $5 \mathrm{~N} 1$ and $9 \mathrm{~N} 2$, increases heterogeneity in NP sizes and disorder in their arrangement. These different factors will be addressed in more detail below through calculations using the GMT. Overall, the extinction spectra of the three chains in Fig. 3 show that substituting large NPs with smaller NPs to create chains of the same length and width yields a lowest energy mode of nearly the same energy, but the composition clearly influences the shape of the spectrum in terms of linewidths and relative intensities of the superradiant mode compared to higher order coupled plasmon modes.

Fig. 3 demonstrates that, at a length corresponding to the infinite chain limit for the chains of largest NPs, changing the composition to smaller NPs while preserving the overall dimensions of the chain will modify the width and intensity but not the energy of the superradiant mode. The question remains, however, if $L_{T o t}$ is the key parameter that determines 
$\lambda_{\max }$ of the superradiant mode, especially considering how $N_{L}$ of chain $20 \mathrm{~N} 4$ exceeds the infinite chain limit (as also verified below by GMT calculations). Fig. 4 addresses this question by comparing chains with the same $N_{L}$ : a chain of 9 single $94 \mathrm{~nm}$ Au NPs (chain 9N1), the same chain of 9 dimers of $47 \mathrm{~nm} \mathrm{Au} \mathrm{NPs} \mathrm{examined} \mathrm{in} \mathrm{Fig.} 3$ (chain 9N2), and a chain of 9 linear tetramers of $25 \mathrm{~nm} \mathrm{Au} \mathrm{NPs} \mathrm{(chain} \mathrm{9N4).} \mathrm{Note} \mathrm{that} \mathrm{when} \mathrm{substituting} \mathrm{the} \mathrm{large} 94 \mathrm{~nm}$ Au NPs with dimers of $47 \mathrm{~nm}$ Au NPs, preserving $N_{L}$, causes $L_{T o t}$ to change by a factor of two, and so forth with tetramers of the $25 \mathrm{~nm}$ Au NPs, while still keeping the width the same.

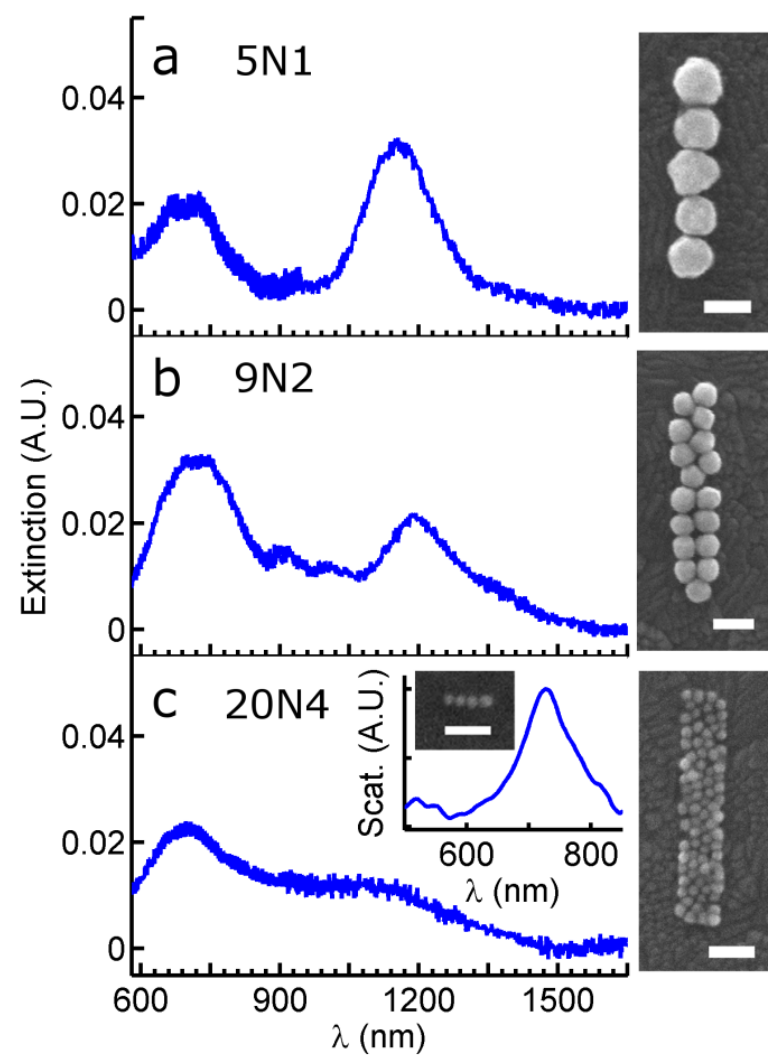

Fig. 3 Extinction spectra of chains having the same $L_{T o t}$ and width and their corresponding SEM images. Extinction spectra for (a) chain $5 \mathrm{~N} 1$ with $\mathrm{d}=94 \mathrm{~nm}$, (b) chain $9 \mathrm{~N} 2$ with $d=47 \mathrm{~nm}$, and (c) chain $20 \mathrm{~N} 4$ with $d=25 \mathrm{~nm}$. The inset of (c) shows the scattering spectrum measured for the repeat unit of chain $20 \mathrm{~N} 4$, the linear tetramer. The scale bars in the SEM images represent $100 \mathrm{~nm}$. The data in (b) are reproduced from figure 3(b) in reference 14 .

Interestingly, the lowest energy resonances in the extinction spectra of chains 9N1, 9N2, and 9N4 occur again at almost the same wavelengths of $1200 \mathrm{~nm}, 1190 \mathrm{~nm}$, and $1170 \mathrm{~nm}$, 
respectively (Fig. 4). Additional extinction spectra of the chains studied here are provided in Figure S6 to show the invariance of the excitation energy of the lowest energy mode. This result indicates that $N_{L}$, and not $L_{T o t}$, determines the energy of the lowest energy resonance. This conclusion is further supported by examining Figs. 3 and 4 in parallel. Nearly doubling $L_{T o t}$ between chain $5 \mathrm{~N} 1$ to chain $9 \mathrm{~N} 1$, leads to a minorant redshift of $\sim 40 \mathrm{~nm}$. More significantly, reducing $L_{T o t}$ by 4 times from chain $9 \mathrm{~N} 1$ to chain $9 \mathrm{~N} 4$ leads to a small blueshift of $30 \mathrm{~nm}$. The experimental data of Figs. 3 and 4 thus demonstrate that $N_{L}$ is the more important geometric parameter that determines the energy of the lowest energy mode.

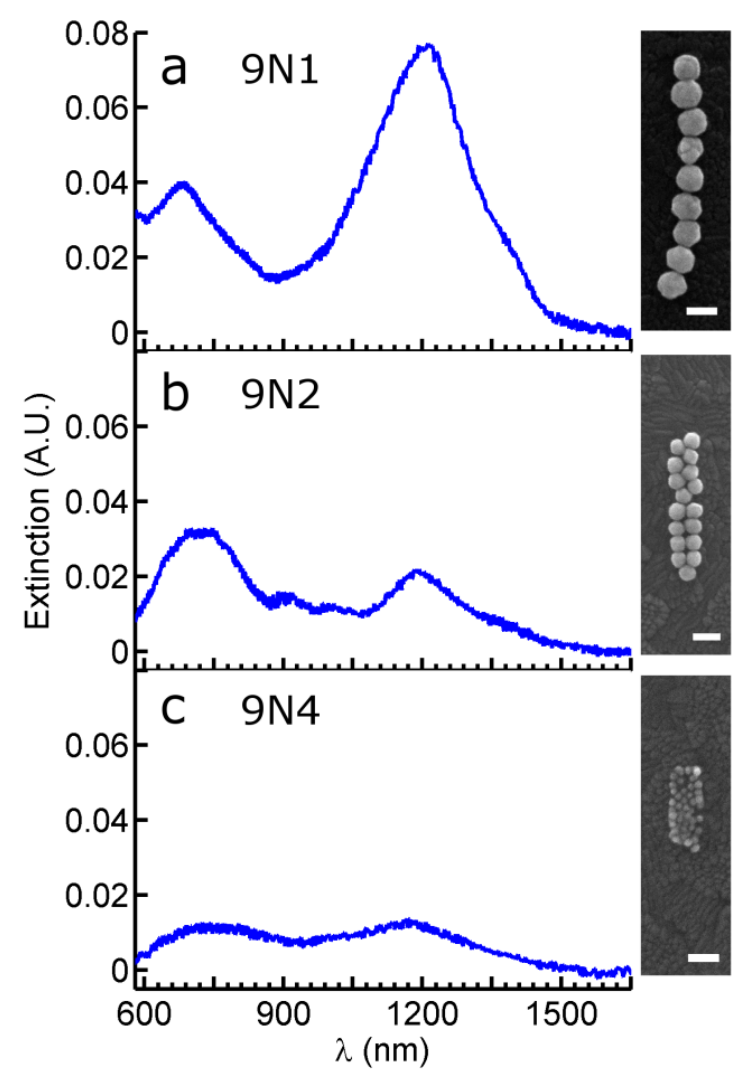

Fig. 4 Extinction spectra of chains having approximately the same number of monomers and their corresponding SEM images. (a) chain $9 \mathrm{~N} 1$ with $d=94 \mathrm{~nm}$, (b) chain $9 \mathrm{~N} 2$ with $d=47 \mathrm{~nm}$, and (c) chain $9 \mathrm{~N} 4$ with $d=25 \mathrm{~nm}$. The scale bars represent $100 \mathrm{~nm}$. The data in (b) are reproduced from figure 3(b) in reference 14. 
Simulations via the GMT give powerful insight into the identity of the resonant modes and the physical mechanisms leading to the shape of the spectra. Furthermore, only theory can provide a systematic comparison of how realistic systems differ from perfectly ordered plasmonic polymers. Although other gridded numerical approaches such as finite-difference time domain (FDTD) method and finite-element method (FEM) are able to simulate objects with arbitrary shapes, the size and fineness of the gridding required for the large sizes and subnanometer gaps of the chains studied here make these methods too computationally expensive. The analytical approach using the GMT, though still computationally expensive, is in fact an affordable theoretical tool. The GMT simulations considered 23 multipole modes of the individual Au NPs to achieve convergence and inherently considered plasmon coupling, higher order modes, superradiance, and retardation effects, which were discussed qualitatively above. Spectra and charge distributions were at the lowest energy resonance simulated for well-ordered chains (5a-c, 6a-c) and experimental chains in Figs. 3 and 4 (5d-f, 6d-f). 

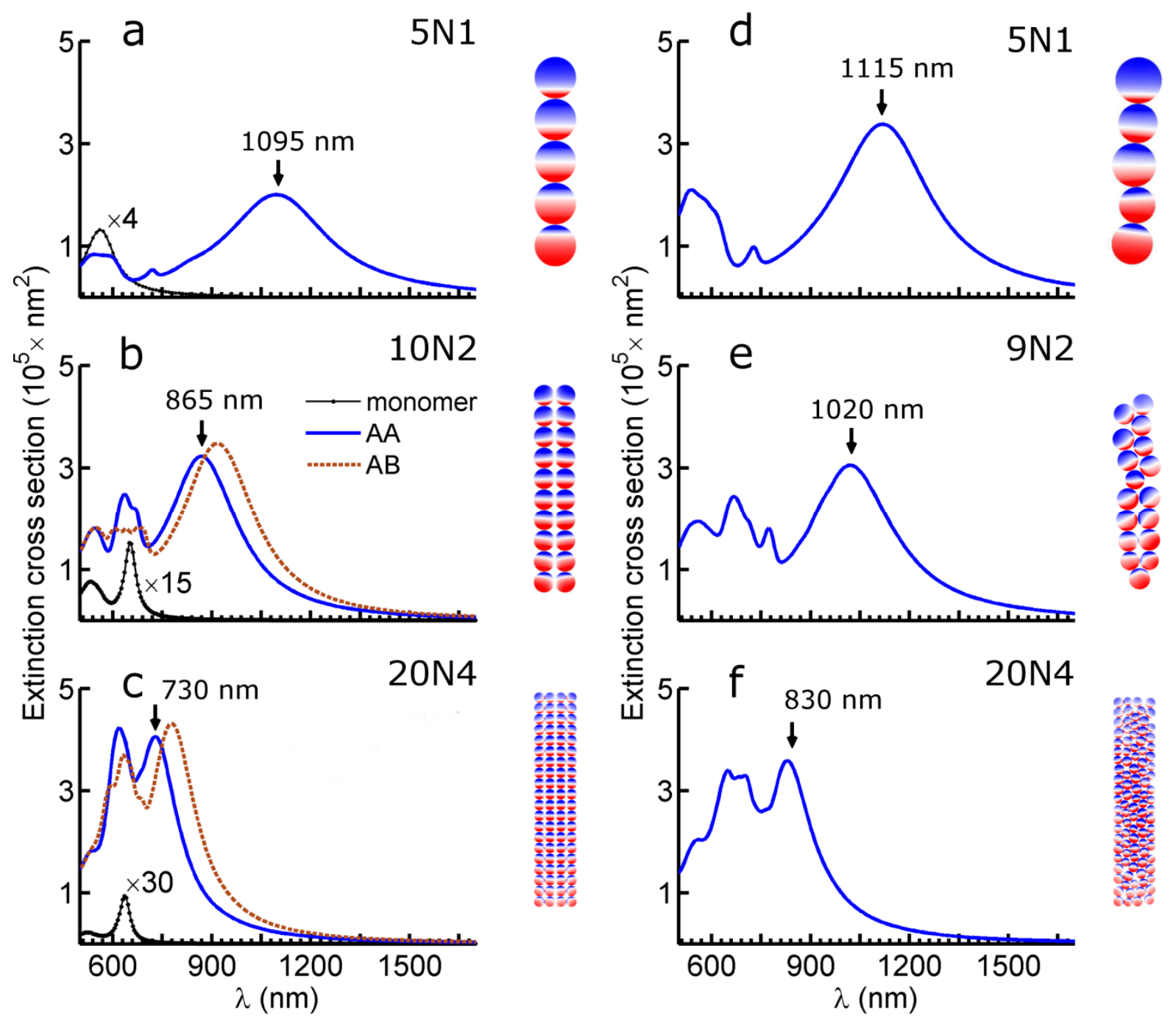

Fig. 5 Extinction spectra and charge distributions at the lowest energy peak calculated via the GMT for ideal (a-c) and experimental (d-f) plasmonic polymers of Au NPs with the same $L_{\text {Tot }}$ and width. The modeled chains comprise $94 \mathrm{~nm} \mathrm{Au}$ NPs (a) \& (d), $47 \mathrm{~nm}$ Au NPs (b) \& (e), and $25 \mathrm{~nm}$ Au NPs (c) \& (f). The black lines in (a-c) give the simulated extinction spectra of the monomer repeat units: (a) a single $94 \mathrm{~nm}$ Au NP, (b) a dimer of $47 \mathrm{~nm} \mathrm{Au} \mathrm{NPs,}$ and (c) a linear tetramer of $25 \mathrm{~nm}$ Au NPs. (b) and (c) show calculated spectra for both AA-packed (solid blue lines) and AB-packed (dotted brown lines) configurations. The schematic drawings of the AA-packed and ABpacked chains are shown in Fig. S5. The spectrum in (f) was calculated for a randomized arrangement of $25 \mathrm{~nm} \mathrm{Au}$ NPs. Enlarged views of the charge distributions for Figs. 5 and 6 are provided in Supplementary Information Fig. S4. It is noted that the $N_{L}$ of the ideal chain $10 \mathrm{~N} 2$ is chosen to compare with the ideal plasmonic polymers of other repeat units to the same $L_{T o t}$; therefore $10 \mathrm{~N} 2$ is not meant to directly compare with the experimental structure $9 \mathrm{~N} 2$.

In Fig. 5a-b, the calculated extinction spectra of the well-ordered counterparts of $5 \mathrm{~N} 1$ and 10N2 in Fig. 3 (blue lines) share qualitatively similar line shapes. Both the experiments and simulations reveal significant redshifting of the superradiant chain mode compared to the monomer repeat units (black lines). The largest redshift occurs for chain 5N1 (Fig. 5a), with a 
superradiant mode at $1095 \mathrm{~nm}$, which is shifted by $230 \mathrm{~nm}$ relative to the same mode of chain 10N2 (Fig. 5b), the well-ordered version of chain 9N2. After the disorder of the particle size and position is accounted for in Fig. 5d-e, the difference in the energy of the superradiant mode between $5 \mathrm{~N} 1$ and $9 \mathrm{~N} 2$ becomes smaller, improving agreement between experiment and theory. The improvement is due to the fact that disorder tends to maximize interparticle plasmon coupling especially in a several NP wide chain and therefore redshifts the superradiant mode. This rationale can be verified by considering non-close vs close packing of the ideal chains (Fig. $5 b-c)$. The extinction spectra of the hexagonally close packed versions (AB, dashed brown lines in Figs. 5b-c) of chains $10 \mathrm{~N} 2$ and 20N4 have superradiant peaks that are further redshifted and broadened from the square (AA) packed configurations. The $\mathrm{AB}$ configuration alleviates some of the repulsive plasmonic interactions along the transverse chain axis, thereby lowering the energy of the superradiant mode. Furthermore, as seen from Fig. 5d-f, introducing additional degrees of disorder through different NP sizes, shapes, and positions causes the superradiant mode of disordered structures to redshift further than those of the well-ordered counterparts; disorder likely allows NPs to pack more closely in some regions of chains 9N2 and 20N4 and hence couple more strongly through near-field interactions. Small differences do exist, however, between the theory and the experiment for the $5 \mathrm{~N} 1$ and $9 \mathrm{~N} 2$ chains, likely because of the inhomogeneity of substrate and the faceted shapes of real NPs.

For chain 20N4, which contains the smallest NPs, the simulation (Fig. 5c) cannot produce the peak at $1200 \mathrm{~nm}$ seen in the experiment even for the totally randomized structure (Fig. 5f). This discrepancy is attributed to the fact that the input structure in the simulation is limited to the resolution of the SEM, which is not capable of exactly determining the real spacing and size of 
the smallest NPs. Therefore we cannot rule out the possibility of touching NPs in the structure and this effect will be discussed in details below.

Similar to the results in Fig. 5, disorder has only a small effect on the position of the lowest energy resonance maximum for the chain composed of a single column of $94 \mathrm{~nm} \mathrm{Au} \mathrm{NPs}$ (Figs. 6a and 6d). The resonance maximum and spectral shape of the simulated spectra are in good agreement with the experimental results (Fig. 4a). Again, the simulated spectra of AA- and AB- packed chains in Fig. 6b-c again confirm that AB- packing, with smaller interstitial spaces and weaker repulsive interactions, allow the chain modes to further redshift and broaden compared to AA-packing. This effect is again amplified in Fig. 6d-f for the smallest NPs, where a distribution of sizes and more disordered packing promote plasmon coupling and further lower the energies of the superradiant modes. Note that the spectra for the medium size NP chains are the same as in Fig. 5.

By considering chains with ideal configurations, we can furthermore test the conclusion that $N_{L}$ is a more important parameter than $L_{T o t}$ in determining the when the excitation energy of the superradiant mode saturates, or when the onset of the infinite chain limit is reached. In the inset of Fig. 6c the normalized wavelength shift of the lowest energy mode is plotted for ideal AA packed chains as a function of $N_{L}$ for the three NP sizes. Chains 3N1, 6N2 and 12N4, indicated by the arrows, have approximately the same length and width but different $N_{L}$. It is clear that the redshift for chain $6 \mathrm{~N} 2$ and $12 \mathrm{~N} 4$ has already saturated while for chain $3 \mathrm{~N} 1$ it has not. This comparison demonstrates that $N_{L}$ and not $L_{T o t}$ determines the onset of the infinite chain limit. Once the infinite chain limit is reached for each NP size, the redshift of the superradiant mode becomes negligible and the total chain length $L_{T o t}$ is not important. 


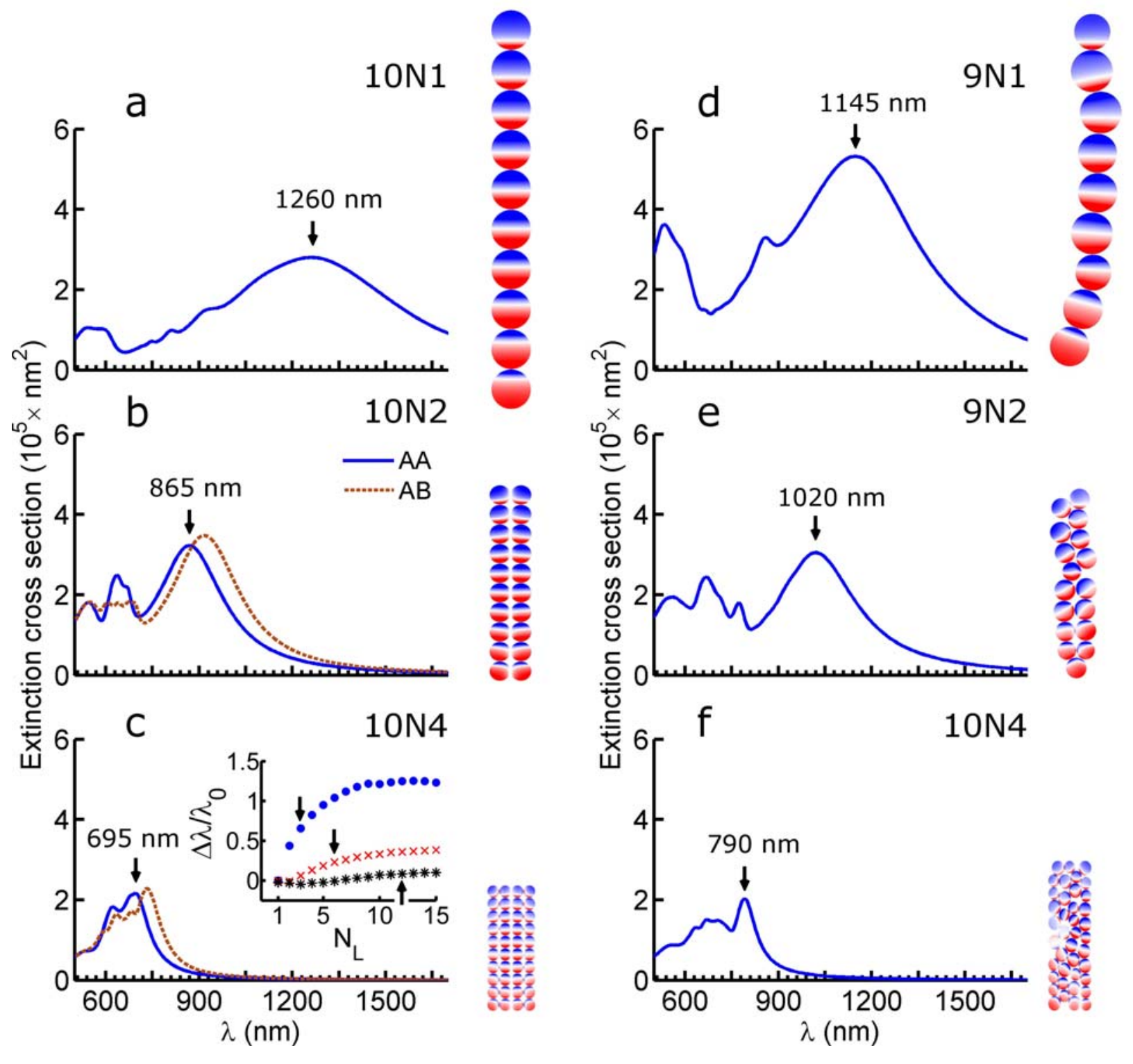

Fig. 6 Extinction spectra and charge distributions at the lowest energy peak calculated via the GMT for ideal (a-c) and experimental (d-f) plasmonic polymers of Au NPs with the same $N_{L}$ and width. The modeled chains comprise $94 \mathrm{~nm}$ Au NPs (a) \& (d), $47 \mathrm{~nm}$ Au NPs (b) \& (e), and $25 \mathrm{~nm}$ Au NPs (c) \& (f). (b) and (c) show spectra for both AA-packed (solid blue lines) and AB-packed (dotted brown lines) configurations. The inset of (c) gives the relative shift of the lowest energy peak with increasing $N_{L}$ for monomers of individual $94 \mathrm{~nm}$ Au NPs (blue dots), dimers of $47 \mathrm{~nm} \mathrm{Au} \mathrm{NPs} \mathrm{(red} \mathrm{crosses),} \mathrm{and} \mathrm{linear} \mathrm{tetramers} \mathrm{of} 25 \mathrm{~nm}$ Au NPs (black asterisks). The arrows in the inset indicate the $3 \mathrm{~N} 1,6 \mathrm{~N} 2$ and $12 \mathrm{~N} 4$ chains, respectively. The spectrum in (f) was calculated for a randomized arrangement of $25 \mathrm{~nm} \mathrm{Au}$ NPs. It is also noted that the $N_{L}$ of the ideal chain $10 \mathrm{~N} 1$ or $10 \mathrm{~N} 2$ is chosen to compare with the ideal plasmonic polymers of other repeat units to the same $N_{L}$; therefore neither $10 \mathrm{~N} 1$ nor $10 \mathrm{~N} 2$ is meant to directly compare with their experimental counterparts. 
Despite the good agreement between experiment and theory for the chains composed of the large and medium size NPs, we cannot ignore the contrast between the measured spectra for chains 20N4 and 9N4 in Figs. 3c and 4c, where the lowest energy peaks of all structures measured occur near $1200 \mathrm{~nm}$, and the simulations of Figs. $5 \mathrm{f}$ and $6 \mathrm{f}$, where even the disordered arrangement does not redshift the lowest energy peak beyond $830 \mathrm{~nm}$. The discussion of the experimental chains $20 \mathrm{~N} 4$ and 9N4 acknowledged that the resolution of the SEM images does not allow us to rule out conductive interactions. The inability to redshift the lowest energy peaks of these chains past $900 \mathrm{~nm}$ with solely capacitive interactions at minimum separations of $0.5 \mathrm{~nm}$ in Figs. 5 and 6 further implicate contributions from conductive interactions to the spectral intensity at $1200 \mathrm{~nm}$ in Figs. 3c and 4c.

Metallic contact is, however, not solely responsible for the lowest energy peaks of 20N4 and 9N4 in Figs. 3c and 4c, respectively. This conclusion is derived from intentionally fusing NPs in a chain composed of $25 \mathrm{~nm}$ NPs due to prolonged exposure to high intensity lamp light (Fig. S3). The lowest energy mode now appears even further redshifted at $1350 \mathrm{~nm}$ in the measured spectrum of the fused chain in Fig. S3a, which has the same dimensions as chain 20N4. Similarly, the intensity of the peak at $1200 \mathrm{~nm}$ in Fig. S3b is enhanced for this fused version of chain 9N4. Furthermore, these lowest energy peaks of the fused chains are narrower than those of chains 20N4 and 9N4 in Figs. 3c and 4c. These redshifts and linewidth narrowing are consistent with charge transfer plasmons. ${ }^{74}$ These observations collectively indicate that plasmon modes besides those arising from metallic contact contribute to the lowest energy peaks for the experimental chains 20N4 and 9N4 in Figs. 3c and 4c.

It is expected that the assemblies of smallest NPs are more likely to sustain plasmon modes arising from conductive contact and electron tunneling than assemblies of the other two 
NP sizes for two reasons. First, the $25 \mathrm{~nm}$ NPs likely experience the least electrostatic repulsion during assembly considering they have the least charged surfaces among the NPs used here, revealed by zeta potential measurements (Table S1). The reduced charges on the $25 \mathrm{~nm}$ Au NPs likely subject their assemblies to interparticle separations $<0.5 \mathrm{~nm}$, where electron tunneling is expected to occur. ${ }^{27,38,43,62}$ Second, as the diameter of the NP shrinks, quantum tunneling of electrons between NPs due to the 'spill-out' of the electron cloud sets in at larger separations relative to their diameters. ${ }^{75}$ Considering a dimer of NPs, metallic contact between the two NPs imparts a narrow low energy peak, a charge transfer plasmon, resembling that of a continuous structure, while tunneling between closely separated but non-touching NPs presents an additional dissipation pathway for the bonding dipolar plasmon. ${ }^{38,76,77}$ To summarize recent literature, Dionne et al. experimentally quantified the onset of quantum tunneling between Ag NPs with diameters of $9 \mathrm{~nm}$ at an edge-to-edge separation of $1 \mathrm{~nm} \cdot{ }^{38}$ Baumberg and Aizpurua have likewise experimentally and provided general quantum corrected models for the onset of quantum tunneling at gaps of $\sim 0.4 \mathrm{~nm}$ for Au NPs with diameters of $50 \mathrm{~nm}^{62,75}$ A rigorous analysis of how the onsert of quantum tunneling between NPs is determined by their diameters is beyond the scope of these and our current study but remains an important topic of active investigation in the field. The additional dissipation pathway provided by tunneling between NPs broadens, blueshifts, and decreases the intensity of the bonding dipolar plasmon. Considering $\sim 80$ NPs in chain $20 \mathrm{~N} 4$ and $\sim 40$ NPs in chain $9 \mathrm{~N} 4$, the broad peaks centered around $1200 \mathrm{~nm}$ in Figs. $3 \mathrm{c}$ and $4 \mathrm{c}$ are therefore assigned to a superposition of conductive contact, electron tunneling, and capacitive interactions between many NPs.

Our conclusions regarding the contribution from several coupling mechanisms for chains composed of $25 \mathrm{~nm}$ NPs is furthermore justified by comparing the linewidth of the lowest energy 
resonance in experiments and simulations. Because superradiance is related to the volume of the

structure, ${ }^{66}$ the linewidths of the lowest energy mode in $20 \mathrm{~N} 4$ and $10 \mathrm{~N} 4$ should be narrower than all the other chains. However, only simulations that only consider capacitive effects including superradiance, and not the experiments, agree with this trend. The unexpectedly broad linewidth of the smallest NP chains is therefore indicative of other mechanisms such as tunneling and conductive interactions in the structure, which are not accounted for by the GMT and incorporating them into grid based calculations for the number of NPs considered here is beyond the scope of this study.

\section{CONCLUSIONS}

In conclusion, we have experimentally and theoretically investigated the sensitivity of the optical response of plasmonic polymers on their defining properties, i.e. 1) the existence of an infinite chain limit as evidenced by the saturation of the redshifting of the lowest energy mode; 2) determination of the overall optical response by the identity of the repeat unit; 3) tolerance to disorder in NP size and arrangement. In particular, our comparisons tested the importance of the number of repeat units of the polymer, $N_{L}$, and the total length of the polymer, $L_{T o t}$, on the wavelength of the lowest energy peak when the width of the polymer was conserved by replacing a large NP with a dimer of medium NPs or linear quadrumer of small NPs. We find that $N_{L}$, and not $L_{T o t}$, is the more important parameter in determining the energy of the superradiant mode and the onset of the infinite chain limit. Our combined experimental and theoretical investigations confirm that, for the relatively large chains investigated here, the wavelengths of the collective chain resonances are determined by both plasmon coupling and 
retardation. The overall intensities of the extinction spectra increased with increasing NP size, resulting from increasing volumes of Au despite similar chain widths and lengths. The spectral linewidths, especially of the lowest energy mode, were determined by multiple pathways that dephase the plasmon resonance including dynamic depolarization, radiative damping, depolarization due to capacitive plasmon coupling, and likely electron tunneling for the chains composed of the smallest NPs. Our study therefore further defines the role of the repeat unit in plasmonic polymers. In particular, the number of repeat units determines the wavelength of the lowest energy mode, but the identity of the repeat unit, and hence the composition of the plasmonic polymer, tunes the shape of the spectrum.

\section{ASSOCIATED CONTENT}

Supplementary Information: Au NP size distributions, simulations demonstrating how the medium refractive index was determined, zeta potential measurements, spectra of partially fused chains of $25 \mathrm{~nm} \mathrm{Au} \mathrm{NPs,} \mathrm{enlarged} \mathrm{views} \mathrm{of} \mathrm{the} \mathrm{charge} \mathrm{distributions} \mathrm{from} \mathrm{Figs.} 5$ and 6, and schematic drawings of AA-packed and AB-packed chains.

Notes: The authors declare no competing financial interests.

\section{ACKNOWLEDGMENT}

This work was funded by the Robert A. Welch Foundation (C-1664), ONR (N00014-10-1-0989), and NSF (CHE-0955286). J.O. was supported by an NSF Graduate Research Fellowship Grant 
No. (0940902) and P.S. acknowledges support from the Royal Thai Government. L.S.S. thanks David Solis and Wei-Shun Chang for assistance with the optical measurements. We thank Christy Landes, Peter Nordlander, and Naomi Halas for insightful discussions and preparation of this manuscript. 


\section{REFERENCES}

1. K. H. Su, Q. H. Wei, X. Zhang, J. J. Mock, D. R. Smith and S. Schultz, Nano Lett., 2003, 3, 1087-1090.

2. J. A. Fan, C. Wu, K. Bao, J. Bao, R. Bardhan, N. J. Halas, V. N. Manoharan, P. Nordlander, G. Shvets and F. Capasso, Science, 2010, 328, 1135-1138.

3. T. Chen, M. Pourmand, A. Feizpour, B. Cushman and B. M. Reinhard, J. Phys. Chem. Lett., 2013, 4, 2147-2152.

4. L. V. Brown, H. Sobhani, J. B. Lassiter, P. Nordlander and N. J. Halas, ACS Nano, 2010, 4, 819-832.

5. M. Chandra, A.-M. Dowgiallo and K. L. Knappenberger, Jr., J. Am. Chem. Soc., 2010, 132, 15782-15789.

6. A. M. Funston, C. Novo, T. J. Davis and P. Mulvaney, Nano Lett., 2009, 9, 1651-1658.

7. L. Chuntonov and G. Haran, Nano Lett., 2013, 13, 1285-1290.

8. D. W. Brandl, N. A. Mirin and P. Nordlander, J. Phys. Chem. B, 2006, 110, 1230212310.

9. N. W. Bigelow, A. Vaschillo, J. P. Camden and D. J. Masiello, ACS Nano, 2013, 7, 45114519 .

10. J. J. Choquette, K.-P. Marzlin and B. C. Sanders, Phys. Rev. A: At., Mol., Opt. Phys., 2010, 82, 023827.

11. K. Liu, Z. Nie, N. Zhao, W. Li, M. Rubinstein and E. Kumacheva, Science, 2010, 329, 197-200.

12. A. Lukach, K. Liu, H. Therien-Aubin and E. Kumacheva, J. Am. Chem. Soc., 2012, 134, 18853-18859.

13. A. Klinkova, H. Thérien-Aubin, R. M. Choueiri, M. Rubinstein and E. Kumacheva, Proc. Natl. Acad. Sci. U. S. A., 2013, 110, 18775-18779.

14. L. S. Slaughter, B. A. Willingham, W.-S. Chang, M. H. Chester, N. Ogden and S. Link, Nano Lett., 2012, 12, 3967-3972.

15. K. Liu, A. Ahmed, S. Chung, K. Sugikawa, G. Wu, Z. Nie, R. Gordon and E. Kumacheva, ACS Nano, 2013, 7, 5901-5910.

16. K. Ueno, S. Juodkazis, V. Mizeikis, D. Ohnishi, K. Sasaki and H. Misawa, Opt. Express, 2007, 15, 16527-16539.

17. P. F. Barbara, A. J. Gesquiere, S.-J. Park and Y. J. Lee, Acc. Chem. Res., 2005, 38, 602610. 
18. G. D. Scholes and G. Rumbles, Nat. Mater., 2006, 5, 683-696.

19. G. Padmanaban and S. Ramakrishnan, J. Am. Chem. Soc., 2000, 122, 2244-2251.

20. D. A. Vanden Bout, W.-T. Yip, D. Hu, D.-K. Fu, T. M. Swager and P. F. Barbara, Science, 1997, 277, 1074-1077.

21. B. Willingham and S. Link, Opt. Express, 2011, 19, 6450-6461.

22. P. K. Jain and M. A. El-Sayed, J. Phys. Chem. C, 2008, 112, 4954-4960.

23. M. Quinten and U. Kreibig, Appl. Opt., 1993, 32, 6173-6182.

24. J. M. Gérardy and M. Ausloos, Phys. Rev. B: Condens. Matter Mater. Phys., 1982, 25, 4204-4229.

25. S. J. Barrow, A. M. Funston, D. E. Gómez, T. J. Davis and P. Mulvaney, Nano Lett., 2011, 11, 4180-4187.

26. W.-S. Chang, L. S. Slaughter, B. P. Khanal, P. Manna, E. R. Zubarev and S. Link, Nano Lett., 2009, 9, 1152-1157.

27. R. Esteban, R. W. Taylor, J. J. Baumberg and J. Aizpurua, Langmuir, 2012, 28, 88818890.

28. T. Shimada, K. Imura, H. Okamoto and M. Kitajima, Phys. Chem. Chem. Phys., 2013, 15, 4265-4269.

29. F. Hide, M. A. Díaz-García, B. J. Schwartz, M. R. Andersson, Q. Pei and A. J. Heeger, Science, 1996, 273, 1833-1836.

30. J. Vogelsang and J. M. Lupton, J. Phys. Chem. Lett., 2012, 3, 1503-1513.

31. R. H. Friend, R. W. Gymer, A. B. Holmes, J. H. Burroughes, R. N. Marks, C. Taliani, D. D. C. Bradley, D. A. D. Santos, J. L. Bredas, M. Logdlund and W. R. Salaneck, Nature, 1999, 397, 121-128.

32. S.-C. Yang, H. Kobori, C.-L. He, M.-H. Lin, H.-Y. Chen, C. Li, M. Kanehara, T. Teranishi and S. Gwo, Nano Lett., 2010, 10, 632-637.

33. L. S. Slaughter, Y. Wu, B. A. Willingham, P. Nordlander and S. Link, ACS Nano, 2010, 4, 4657-4666.

34. P. Nordlander, C. Oubre, E. Prodan, K. Li and M. I. Stockman, Nano Lett., 2004, 4, 899903.

35. C. Pecharroman, Phys. Chem. Chem. Phys., 2009, 11, 5922-5929.

36. L. Slaughter, W.-S. Chang and S. Link, J. Phys. Chem. Lett., 2011, 2, 2015-2023.

37. D.-S. Kim, J. Heo, S.-H. Ahn, S. W. Han, W. S. Yun and Z. H. Kim, Nano Lett., 2009, 9, 3619-3625. 
38. J. A. Scholl, A. García-Etxarri, A. L. Koh and J. A. Dionne, Nano Lett., 2012, 13, 564569.

39. S. N. Sheikholeslami, A. García-Etxarri and J. A. Dionne, Nano Lett., 2011, 11, 39273934.

40. B. Yan, S. V. Boriskina and B. r. M. Reinhard, J. Phys. Chem. C, 2011, 115, 4578-4583.

41. N. Liu, S. Mukherjee, K. Bao, L. V. Brown, J. Dorfmüller, P. Nordlander and N. J. Halas, Nano Lett., 2011, 12, 364-369.

42. L. Chuntonov and G. Haran, J. Phys. Chem. C, 2011, 115, 19488-19495.

43. L. Jiang, W. C. Wang, H. Fuchs and L. F. Chi, Small, 2009, 5, 2819-2822.

44. Y. Cui, M. T. Bjork, J. A. Liddle, C. Sonnichsen, B. Boussert and A. P. Alivisatos, Nano Lett., 2004, 4, 1093-1098.

45. A. Guerrero-Martínez, M. Grzelczak and L. M. Liz-Marzán, ACS Nano, 2012, 6, 36553662 .

46. D. Solis, B. Willingham, S. L. Nauert, L. S. Slaughter, J. Olson, P. Swanglap, A. Paul, W.-S. Chang and S. Link, Nano Lett., 2012, 12, 1349-1353.

47. S. A. Maier, P. G. Kik and H. A. Atwater, Appl. Phys. Lett., 2002, 81, 1714-1716.

48. L. A. Sweatlock, S. A. Maier, H. A. Atwater, J. J. Penninkhof and A. Polman, Phys. Rev. B: Condens. Matter Mater. Phys., 2005, 71, 235408.

49. A. F. Koenderink, R. de Waele, J. C. Prangsma and A. Polman, Phys. Rev. B: Condens. Matter Mater. Phys., 2007, 76, 201403-201404.

50. N. Harris, M. D. Arnold, M. G. Blaber and M. J. Ford, J. Phys. Chem. C, 2009, 113, 2784-2791.

51. L. Zhao, K. L. Kelly and G. C. Schatz, J. Phys. Chem. B, 2003, 107, 7343-7350.

52. D. S. Citrin, Nano Lett., 2005, 5, 985-989.

53. P. K. Jain, W. Huang and M. A. El-Sayed, Nano Lett., 2007, 7, 2080-2088.

54. S. Marhaba, G. Bachelier, C. Bonnet, M. Broyer, E. Cottancin, N. Grillet, J. Lermé, J.-L. Vialle and M. Pellarin, J. Phys. Chem. C, 2009, 113, 4349-4356.

55. I. Romero, J. Aizpurua, G. W. Bryant and F. J. Garcia de Abajo, Opt. Express, 2006, 14, 9988-9999.

56. M. S. Scheurer, M. D. Arnold, J. Setiadi and M. J. Ford, J. Phys. Chem. C, 2011, 116, 1335-1343.

57. F. Rüting, Phys. Rev. B: Condens. Matter Mater. Phys., 2011, 83, 115447. 
58. L. S. Slaughter, W.-S. Chang, P. Swanglap, A. Tcherniak, B. P. Khanal, E. R. Zubarev and S. Link, J. Phys. Chem. C, 2010, 114, 4934-4938.

59. D. W. Mackowski and M. I. Mishchenko, J. Quant. Spectrosc. Radiat. Transfer, 2011, 112, 2182-2192.

60. J. M. Gérardy and M. Ausloos, Phys. Rev. B: Condens. Matter Mater. Phys., 1984, 30, 2167.

61. T. Peng, A. Balijepalli, S. K. Gupta and T. LeBrun, J. Comput. Inf. Sci. Eng., 2007, 7, 330-338.

62. K. J. Savage, M. M. Hawkeye, R. Esteban, A. G. Borisov, J. Aizpurua and J. J.

Baumberg, Nature, 2012, 491, 574-577.

63. B. D. Lubachevsky, F. H. Stillinger and E. N. Pinson, J. Stat. Phys., 1991, 64, 501-524.

64. B. D. Lubachevsky and F. H. Stillinger, J. Stat. Phys., 1990, 60, 561-583.

65. P. B. Johnson and R. W. Christy, Phys. Rev. B: Condens. Matter Mater. Phys., 1972, 6, 4370-4379.

66. R. H. Dicke, Physical Review, 1954, 93, 99-110.

67. A. F. Koenderink and A. Polman, Phys. Rev. B: Condens. Matter Mater. Phys., 2006, 74, 033402 .

68. S. Zou and G. C. Schatz, Nanotechnology, 2006, 17, 2813.

69. M. Meier and A. Wokaun, Opt. Lett., 1983, 8, 581-583.

70. J. C. Heckel and G. Chumanov, J. Phys. Chem. C, 2011, 115, 7261-7269.

71. R. Esteban, R. Vogelgesang, J. Dorfmuller, A. Dmitriev, C. Rockstuhl, C. Etrich and K. Kern, Nano Lett., 2008, 8, 3155-3159.

72. A. Tcherniak, J. W. Ha, S. Dominguez-Medina, L. S. Slaughter and S. Link, Nano Lett., 2010, 10, 1398-1404.

73. M. K. Kinnan and G. Chumanov, J. Phys. Chem. C, 2010, 114, 7496-7501.

74. Y. Wang, Z. Li, K. Zhao, A. Sobhani, X. Zhu, Z. Fang and N. J. Halas, Nanoscale, 2013.

75. R. Esteban, A. G. Borisov, P. Nordlander and J. Aizpurua, Nature Communications, 2012, 3, 825.

76. P. Song, S. Meng, P. Nordlander and S. Gao, Phys. Rev. B: Condens. Matter Mater. Phys., 2012, 86, 121410.

77. J. Zuloaga, E. Prodan and P. Nordlander, Nano Lett., 2009, 9, 887-891. 
